G protein-mediated signals may be useful. Agents with pathway-selective activities might represent a potentially new generation of drugs. More studies that elucidate the physiologic roles of novel 7TMR signaling mechanisms, such as those reported here by Zhai et al. (3), will be necessary to lay the foundation for such developments.

Address correspondence to: Robert J. Lefkowitz, Howard Hughes Medical Institute, Duke University Medical Center, DUMC 3821, 467 CARL Building, Research Drive, Durham, North Carolina 27710, USA. Phone: (919) 684-2974; Fax: (919) 684-8875; E-mail: lefko001@receptor-biol.duke.edu.

1. Rockman, H., Koch, W., and Lefkowitz, R. 2002 Seven-transmembrane-spanning receptors and heart function. Nature. 415:206-212.

2. Hall, R., Premont, R., and Lefkowitz, R. 1999. Heptahelical receptor signaling: beyond the $\mathrm{G}$ protein paradigm. J. Cell Biol. 145:927-932.

3. Zhai, P., et al. 2005. Cardiac-specific overexpression of AT1 receptor mutant lacking $G_{\alpha} \mathrm{q} / \mathrm{G}_{\alpha} \mathrm{i}$ coupling causes hypertrophy and bradycardia in transgenic mice. J. Clin. Invest. 115:3045-3056. doi:10.1172/ JCI25330.

4. Seta, K., Nanamori, M., Modrall, J., Neubig, R., and Sadoshima, J. 2002. AT1 receptor mutant lacking heterotrimeric $G$ protein coupling activates the Src-Ras-ERK pathway without nuclear translocation of ERKs. J. Biol. Chem. 277:9268-9277.
5. Marrero, M., et al. 1995. Direct stimulation of Jak/ STAT pathway by the angiotensin II AT1 receptor. Nature. 375:247-250.

6. Kohout, T., and Lefkowitz, R. 2003. Regulation of $G$ protein-coupled receptor kinases and arrestins during receptor desensitization. Mol. Pharmacol. 63:9-18.

7. Lefkowitz, R., and Shenoy, S. 2005. Transduction of receptor signals by beta-arrestins. Science. 308:512-517.

8. Herranz, S., et al. 2005. Arrestin-related proteins mediate $\mathrm{pH}$ signaling in fungi. Proc. Natl. Acad. Sci. U. S. A. 102:12141-12146.

9. Kim, J., et al. 2005. Functional antagonism of different $G$ protein-coupled receptor kinases for betaarrestin-mediated angiotensin II receptor signaling. Proc. Natl. Acad. Sci. U. S. A. 102:1442-1447.

10. Luttrell, L., et al. 1999. Beta-arrestin-dependent formation of beta2 adrenergic receptor-Src protein kinase complexes. Science. 283:655-661.

11. DeFea, K., et al. 2000. The proliferative and antiapoptotic effects of substance $P$ are facilitated by formation of a beta-arrestin-dependent scaffolding complex. Proc. Natl. Acad. Sci. U. S. A. 97:11086-11091.

12. Goel, R., Phillips-Mason, P., Raben, D., and Baldassare, J. 2002. alpha-Thrombin induces rapid and sustained Akt phosphorylation by beta-arrestin1dependent and -independent mechanisms, and only the sustained Akt phosphorylation is essential for $\mathrm{G} 1$ phase progression. J. Biol. Chem. 277:18640-18648.

13. Povsic, T., Kohout, T., and Lefkowitz, R. 2003. Beta-arrestin 1 mediates insulin-like growth factor 1 (IGF-1) activation of phosphatidylinositol 3-kinase (PI3K) and anti-apoptosis. J. Biol. Chem. 278:51334-51339.

14. Revankar, C., Vines, C., Cimino, D., and Prossnitz, E. 2004. Arrestins block G protein-coupled receptor- mediated apoptosis. J. Biol. Chem. 279:24578-24584.

15. Gudermann, T., Nurnberg, B., and Schultz, G. 1995. Receptors and $G$ proteins as primary components of transmembrane signal transduction. Part 1. G protein-coupled receptors: structure and function. J. Mol. Med. 73:51-63.

16. Kenakin, T. 2003. Ligand-selective receptor conformations revisited: the promise and the problem. Trends Pharmacol. Sci. 24:346-354.

17. D’Angelo, D., et al. 1997. Transgenic Galphaq overexpression induces cardiac contractile failure in mice. Proc. Natl. Acad. Sci. U. S. A. 94:8121-8126.

18. Esposito, G., et al. 2002. Genetic alterations that inhibit in vivo pressure-overload hypertrophy prevent cardiac dysfunction despite increased wall stress. Circulation. 105:85-92.

19. Akhter, S., et al. 1998. Targeting the receptor-Gq interface to inhibit in vivo pressure overload myocardial hypertrophy. Science. 280:574-577.

20. McDonald, P., et al. 2000. Beta-arrestin2: a receptor-regulated MAPK scaffold for the activation of JNK3. Science. 290:1574-1577.

21. Willoughby, E., and Collins, M. 2005. Dynamic interaction between the dual specificity phosphatase MKP7 and the JNK3 scaffold protein beta-arrestin2. J. Biol. Chem. 280:25651-25658.

22. Hunton, D., et al. 2005. Beta-arrestin2-dependent angiotensin II type $1 \mathrm{~A}$ receptor-mediated pathway of chemotaxis. Mol. Pharmacol. 67:1229-1236.

23. Sun, Y., Cheng, Z., Ma, L., and Pei, G. 2002. Betaarrestin2 is critically involved in CXCR4-mediated chemotaxis, and this is mediated by its enhancement of p38 MAPK activation. J. Biol. Chem. 277:49212-49219.

24. Naga Prasad, S.V., Jayatilleke, A., Madamanchi, A., and Rockman, H.A. 2005. Protein kinase activity of phosphoinositide 3-kinase regulates beta-adrenergic receptor endocytosis. Nat. Cell Biol. 7:785-796.

\title{
Variable phenotypic expression of mutations in genes of the immune system
}

\author{
Rebecca H. Buckley
}

Department of Pediatrics, Duke University Medical Center, Durham, North Carolina, USA.

\begin{abstract}
Discovery of mutated genes that cause various types of primary immunodeficiencies has significantly advanced our understanding of the pathogenesis of these diseases and of the functions of normal gene products. However, it is becoming abundantly clear that the phenotypic presentation of mutations in a given gene can be quite different, depending upon the location and type of mutation but also probably upon other genetic factors and environmental influences. In this issue of the JCI, de Villartay et al. describe a third phenotype for mutations in recombination activating gene 1 (RAG1), in addition to the already known phenotypes of SCID and Omenn syndrome (see the related article on page 3291 ).
\end{abstract}

Nonstandard abbreviations used: ADA, adenosine deaminase; BTK, Bruton tyrosine kinase; CD $3 \varepsilon, C D 3 \varepsilon$ chain; RAG1, recombination activating gene 1; SAP, signaling lymphocyte activation molecule-associated (SLAM-associated) protein; WASP, Wiskott-Aldrich syndrome protein.

Conflict of interest: The author has declared that no conflict of interest exists.

Citation for this article: J. Clin. Invest. 115:2974-2976 (2005). doi:10.1172/JCI26956.
Human primary immunodeficiency diseases have been recognized for only a little over a half century (1). The spectrum of such diseases has grown at an extremely rapid pace during the past 50 years, with currently more than 120 different syndromes having been described (2). For most of that period, the conditions were identified by their clinical and immunologic presentations. How- ever, for the past 12 years, they have been defined extensively at a molecular level. Because making a firm diagnosis by clinical and immunologic criteria has always been problematic due to variability in presentation, it was thought that molecular testing would remove this ambiguity. The report by de Villartay et al. in this issue of the JCI clearly indicates that is not always the case (3). In the pre-molecular diagnostic period, there had been examples within sibships of clinical variability in expression of primary immunodeficiency. One of these was in a family reported by de Saint-Basile et al. (4) in which one sibling had Omenn syndrome and another had SCID. Subsequently, the explanation for this was found when mutations in recombination activating genes 1 and 2 (RAG1 and RAG2, respectively) were found to cause SCID (5), and later hypomorphic 


\section{Table 1}

Mutated immune system genes with variable phenotypic expression

Mutated gene

RAG1

BTK
WASP
SH2D1A

SH2D1A

$C D 3 \varepsilon$
IL2RG

IL2RG

JAK3
Normal gene product

Recombination activating gene 1

Bruton tyrosine kinase
Wiskott-Aldrich syndrome protein
Slam-associated protein (SAP)
$\operatorname{CD} 3 \varepsilon$ chain
Common cytokine receptor $\gamma$ chain $(\gamma c)$
Adenosine deaminase
Jak3

Classic syndrome

$\operatorname{SCID}(4,5,7,15)$

Agammaglobulinemia (8)
Wiskott-Aldrich syndrome (10)
Fatal infectious mononucleosis (11)
$\operatorname{SCID}(14,21)$
$\operatorname{SCID}(15)$
$\operatorname{SCID}(15,17)$
$\operatorname{SCID}(15)$

\section{Variant syndromes}

1. Omenn syndrome $(4,6,7)$

2. Oligoclonal $\gamma / \delta$ T cells, autoimmune disease, and CMV infection (3)

Polysaccharide antibody deficiency (9)

X-linked thrombocytopenia (10)

1. Common variable immunodeficiency (11)

2. Hemophagocytic lymphohistiocytosis (12)

Moderate susceptibility to infection (13)

Moderate combined immunodeficiency (16)

Moderate combined immunodeficiency (17)

Moderate combined immunodeficiency (18)

References are shown in parentheses.

mutations in these genes were found to cause Omenn syndrome (6). It was then found that identical mutations in these genes could cause either syndrome (7). De Villartay and his colleagues (3) now show that another distinct clinical syndrome can be caused by hypomorphic mutations in $R A G$ genes. In contrast to infants with SCID or Omenn syndrome due to RAG mutations, the infants in the current report had autoimmune cytopenias, B cells, normal Ig levels, clonal T cells with $\gamma \delta$ TCRs, normal T cell proliferation, and only slightly elevated NK cell levels. Because all of the infants had disseminated CMV infections, the authors suggest that the expansion of Ti $\delta$ T cells (where Ti represents the TCR expressing the $\gamma \delta$ heterodimer) was due to stimulation by the CMV virus. As the authors point out, there is mounting evidence that T cells with $\gamma \delta$ receptors have a role in host defense against several microbial pathogens, and they have been found to be increased in immunocompromised humans infected with CMV after transplantation. Thus, it is possible that environmental or other genetic factors modify the clinical manifestation of such mutations.

\section{Other genes with phenotypic variability}

Other genes of the immune system in which mutations result in variable phenotypes include those that encode Bruton tyrosine kinase (BTK), Wiskott-Aldrich syndrome protein (WASP), signaling lymphocyte activation molecule-associated (SLAMassociated) protein (SAP), CD3 $\varepsilon$ chain $(\mathrm{CD} 3 \varepsilon)$, common $\gamma$ chain $(\gamma \mathrm{c})$, adenosine deaminase (ADA), and Jak3 (Table 1). These genes encode proteins essential for: (a) B cell development and function
(BTK); (b) T cell development and signaling (CD3e); (c) T cell function and survival (ADA); (d) cytokine receptor signaling ( $\gamma \mathrm{c}$ and Jak3); (e) regulation of cell signaling (SAP); and (f) intracellular signaling and actin polymerization (WASP). Usually, but not always, the phenotypic variability is due to the type and location of the mutation, with hypomorphic mutations causing a less complete immunodeficiency. In the CBA/N mouse, a mutation in the Btk gene results in polysaccharide antibody deficiency, whereas such mutations in humans usually cause agammaglobulinemia (8). However, there is a report of a human with only polysaccharide antibody deficiency who had a mutation in this gene (9). Mutations in the WASP gene result in Wiskott-Aldrich syndrome but also in X-linked thrombocytopenia (10). SH2D1A mutations have been shown to cause variously fatal infectious mononucleosis, common variable immunodeficiency (11), or hemophagocytic lymphohistiocytosis (12). Mutations in CD3 $\varepsilon$ were first reported to cause a relatively mild form of immunodeficiency (13), but more deleterious mutations were later found to cause SCID (14). Mutations in the genes encoding $\gamma \mathrm{c}, \mathrm{ADA}$, and Jak3 usually result in SCID (15) but have also been shown to cause less severe forms of immunodeficiency (16-18).

Clinical ambiguity also arises from the fact that seemingly identical clinical syndromes can be caused by mutations in different immune system genes. For example, it was recently shown that Omenn syndrome can also be caused by mutations in the Artemis gene that encodes a DNA repair factor (19). Other conditions include the hyper-IgM syndrome that can be caused by mutations in at least 6 different genes (20) and SCID, known to be caused by mutations in at least 10 different genes (15, 21). The most striking example of this will likely be the syndrome of common variable immunodeficiency that promises to have many different molecular causes, including mutations in genes that encode SAP (11), inducible costimulator (ICOS) (22), transmembrane activator and calcium modulator and cyclophilin ligand interactor (TACI) (23), and the CD40 ligand (20).

\section{Conclusion}

The above examples are likely only the tip of the iceberg. Discovery of the molecular bases of many primary immunodeficiency diseases has been of major importance in understanding their pathogenesis and inheritance as well as in elucidating the functions of the genes in the immune system. It is hoped that these discoveries will eventually be of importance for gene therapy. The recognition by de Villartay and his colleagues (3) of a new syndrome caused by RAG1 mutations should alert all physicians who care for patients with recurrent infections that atypical presentations may occur when genes of the immune system are mutated. However, from a diagnostic standpoint, until routine molecular testing for genes known to be mutated in primary immunodeficiency is implemented, most of these conditions will go undetected, and the full spectrum of phenotypic and genotypic heterogeneity will not be known. The routine use of molecular testing is unfortunately probably a few years off, considering that it is currently available primarily only in research laboratories and that newborn screening by available immunologic methods is not even performed for any of these conditions. Indeed, there is no screening 
for any of these conditions at any time of life anywhere in the world. Until this happens, there will still be patients who present with recurrent infections who have undiagnosed, genetically determined immunodeficiency, the basis of which is unidentified. Failure to make these diagnoses early in life results in high rates of mortality and morbidity that could be prevented.

Address correspondence to: Rebecca Buckley, Box 2898, Duke University Medical Center, Durham, North Carolina 27710, USA. Phone: (919) 684-2922; Fax: (919) 681-7979; E-mail: buckL003@mc.duke.edu.

\footnotetext{
1. Bruton, O.C. 1952. Agammaglobulinemia. Pediatrics 9:722-728.

2. Notarangelo, L., et al. 2004. Primary immunodeficiency diseases: an update. J. Allergy Clin. Immunol. 114:677-687.

3. de Villartay, J.-P., et al. 2005. A novel immunodeficiency associated with hypomorphic RAG1 mutations and CMV infection. J. Clin. Invest. 115:3291-3299. doi:10.1172/JCI25178.

4. de Saint-Basile, G., et al. 1991. Restricted heterogeneity of T lymphocytes in combined immunodeficiency with hypereosinophilia (Omenn syndrome). J. Clin. Invest. 87:1352-1359.
}

5. Schwarz, K., et al. 1996. RAG mutations in human B cell-negative SCID. Science. 274:97-99.

6. Villa, A., et al. 1998. Partial V(D)J recombination activity leads to Omenn syndrome. Cell. 93:885-896.

7. Corneo, B., et al. 2001. Identical mutations in RAG1 or RAG2 genes leading to defective $\mathrm{V}(\mathrm{D}) \mathrm{J}$ recombinase activity can cause either T-B-severe combined immune deficiency or Omenn syndrome. Blood. 97:2772-2776.

8. Lindvall, J.M., et al. 2005. Bruton's tyrosine kinase: cell biology, sequence conservation, mutation spectrum, siRNA modifications, and expression profiling. Immunol. Rev. 203:200-215.

9. Wood, P.M., et al. 2001. A mutation in Bruton's tyrosine kinase as a cause of selective anti-polysaccharide antibody deficiency. J. Pediatr. 139:148-151.

10. Jin, Y., et al. 2004. Mutations of the Wiskott-Aldrich Syndrome Protein (WASP): hotspots, effect on transcription, and translation and phenotype/genotype correlation. Blood. 104:4010-4019.

11. Morra, M., et al. 2001. Alterations of the X-linked lymphoproliferative disease gene SH2D1A in common variable immunodeficiency syndrome. Blood. 98:1321-1325

12. Arico, M., et al. 2001. Hemophagocytic lymphohistiocytosis due to germline mutations in SH2D1A, the X-linked lymphoproliferative disease gene. Blood. 97:1131-1133.

13. Soudais, C., De Villartay, J.P., Le Deist, F., Fischer, A., and Lisowska-Grospierre, B. 1993. Independent mutations of the human CD3-epsilon gene resulting in a $\mathrm{T}$ cell receptor/CD3 complex immunodeficiency. Nat. Genet. 3:77-81.

14. de Saint Basile, G., et al. 2004. Severe combined immunodeficiency caused by deficiency in either the $\delta$ or the $\varepsilon$ subunit of CD3. J. Clin. Invest 114:1512-1517. doi:10.1172/JCI200422588.

15. Buckley, R.H. 2004. Molecular defects in human severe combined immunodeficiency and approaches to immune reconstitution. Annu. Rev. Immunol. 22:625-655.

16. Schmalstieg, F.C., et al. 1995. Missense mutation in exon 7 of the common $\gamma$ chain gene causes a moderate form of X-linked combined immunodeficiency. J. Clin. Invest. 95:1169-1173.

17. Hershfield, M.S. 2003. Genotype is an important determinant of phenotype in adenosine deaminase deficiency. Curr. Opin. Immunol. 15:571-577.

18. Frucht, D.M., et al. 2001. Unexpected and variable phenotypes in a family with JAK3 deficiency. Genes Immun. 2:422-432.

19. Ege, M., et al. 2005. Omenn syndrome due to ARTEMIS mutations. Blood. 105:4179-4186.

20. Durandy, A., Revy, P., and Fischer, A. 2004. Human models of inherited immunoglobulin class switch recombination and somatic hypermutation defects (hyper-IgM syndromes). Adv. Immunol. 82:295-330.

21. Buckley, R.H. 2004. The multiple causes of human SCID. J. Clin. Invest 114:1409-1411. doi:10.1172/ JCI200423571.

22. Grimbacher, B., et al. 2003. Homozygous loss of ICOS is associated with adult-onset common variable immunodeficiency. Nat. Immunol. 4:261-268.

23. Salzer, U., et al. 2005. Mutations in TNFRSF13B encoding TACI are associated with common variable immunodeficiency in humans. Nat. Genet. 37:820-828.

\title{
New insights into nNOS regulation of vascular homeostasis
}

\author{
Gregg L. Semenza
}

Vascular Biology Program, Institute for Cell Engineering, Departments of Pediatrics, Medicine, Oncology, and Radiation Oncology, and McKusick-Nathans Institute of Genetic Medicine, The Johns Hopkins University School of Medicine, Baltimore, Maryland, USA.

\begin{abstract}
An important physiological response to changes in local or systemic oxygenation is the modulation of vascular tone, which is mediated in part by changes in the activities of the 3 NO synthase (NOS) isoforms. In arterial smooth muscle cells, acute hypoxia induces increased vascular tone, which is attenuated if hypoxia persists. In this issue of the JCI, Ward et al. demonstrate that changes in $\mathrm{O}_{2}$ concentration have effects on neuronal NOS enzymatic activity and gene expression that contribute to vascular homeostasis under conditions of acute and chronic hypoxia (see the related article beginning on page 3128).
\end{abstract}

Every cell in the human body is dependent upon the delivery of adequate concentrations of $\mathrm{O}_{2}$ to maintain normal cellular functions, which are principally powered by ATP derived from mitochondrial oxi-

Nonstandard abbreviations used: HIF-1, hypoxiainducible factor- 1 ; nNOS, neuronal NOS; NOS NO synthase.

Conflict of interest: The author has declared that no conflict of interest exists.

Citation for this article: J. Clin. Invest. 115:2976-2978 (2005). doi:10.1172/JCI26792. dative phosphorylation. The anatomical matching of $\mathrm{O}_{2}$ delivery to demand is determined by the production of secreted factors that stimulate blood vessel growth, most notably VEGF (1). Every step of its biogenesis, from transcription of $V E G F$ gene sequences in the nucleus and protection of the resulting mRNA against degradation to the ribosomal translation and folding of VEGF protein in the endoplasmic reticulum and transport via the Golgi system to the plasma membrane for secretion, is $\mathrm{O}_{2}$ regulated, as is the expression of its cognate receptor on vascular endothelial cells (1-3).

Anatomical responses to changes in $\mathrm{O}_{2}$ demand occur on a scale of days, whereas other physiological responses resulting in alterations in $\mathrm{O}_{2}$ delivery occur on a scale of seconds. Systemic responses are mediated by chemoreceptor cells in the carotid body that depolarize in response to reduced arterial $\mathrm{O}_{2}$ tension, leading to reflex changes in ventilation, heart rate, and vascular tone (4). The vasculature within tissues also responds to acute regional hypoxia by dilation of arterioles that control the flow of blood into each capillary bed, as in the case of increased $\mathrm{O}_{2}$ consumption in skeletal muscle during exercise (5). In contrast, when systemic hypoxia occurs as a result of vascular hypotension (shock), the adrenergic nervous system directs redistribution of blood flow to maintain the perfusion of 